
\section{8} Research Unit, University of Nottingham, Nottingham, UK ${ }^{2}$ Pulmonary Rehabilitation Research Group, Glenfield Hospital, University Hospitals of Leicester NHS Trust, Leicester, UK ${ }^{3}$ Clinical Science Centre University Hospital Aintree, Liverpool, UK

\section{Correspondence to} Dr Charlotte E Bolton, Nottingham Respiratory Research Unit, University of Nottingham, Clinical Sciences, City Hospital Campus, Hucknall Road, Nottingham NG5 1PB, UK; charlotte.bolton@nottingham. ac.uk

Received 29 April 2013 Revised 23 May 2013 Accepted 30 May 2013
To cite: Bolton $C E$, Singh SJ, Walker PP, et al. Thorax 2013;68:887-888.

\title{
Commentary: the British Thoracic Society guideline on pulmonary rehabilitation in adults
}

\author{
Charlotte E Bolton, ${ }^{1}$ Sally J Singh, ${ }^{2}$ Paul P Walker, ${ }^{3}$ on behalf of the British Thoracic \\ Society Pulmonary Rehabilitation Guideline Group
}

\begin{abstract}
The first British Thoracic Society guideline on pulmonary rehabilitation in adults is published in the accompanying Thorax supplement. Focusing on delivery of a quality pulmonary rehabilitation service, the evidenced-based guideline encompasses recommendations on the composition of the service, referral of patients and who benefits, the expected patient-related outcome measures and markers, pulmonary rehabilitation following exacerbations, the post-rehabilitation period and areas requiring further research. In this commentary, we introduce the guideline and discuss pulmonary rehabilitation in the wider context of chronic disease management of respiratory patients.
\end{abstract}

The publication of the first British Thoracic Society (BTS) pulmonary rehabilitation guideline permits an opportunity to reflect and highlight this key therapy for patients with chronic respiratory disease. $^{1}$

Over the years, the BTS has been a strong supporter of pulmonary rehabilitation and the statement published in 2001 has been an invaluable tool in supporting the development and setting up of programmes across the UK. ${ }^{2}$ However, the subsequent decade has led to a greater understanding of pulmonary rehabilitation, necessitating a formal guideline. A rigorous methodology in line with the AGREE collaboration was used, based on PICO (Patient, Intervention, Control, Outcome) style questions set by the multidisciplinary Guideline Development Group, and the literature was robustly critiqued to demonstrate the strength of evidence and develop the subsequent recommendations. We are not aware of pulmonary rehabilitation guidelines that have previously gone through this in-depth methodology.

Although principally targeted at healthcare professionals in the UK, we hope it is of interest to those who work in other healthcare systems. We also believe the guideline is important for those who commission services. Large randomised controlled trials, embraced into Cochrane systematic reviews, have demonstrated the undeniable patientrelated benefits in exercise tolerance, quality of life and psychological status ${ }^{3}$; consequently pulmonary rehabilitation is wholeheartedly recommended in chronic obstructive pulmonary disease (COPD) guidelines. Despite this very strong body of evidence for the benefits of pulmonary rehabilitation in patients with COPD, it still remains easy to overlook the tremendous impact it can have on a patient who is functionally disabled.

The value of pulmonary rehabilitation extends beyond the individual, and although not a component of this guideline, there is evidence to support the cost effectiveness of rehabilitation within the UK healthcare system. ${ }^{4}$ The 'value pyramids' proposed by IMPRESS (http://www.impressresp.com) clearly demonstrate that pulmonary rehabilitation represents real value for money as an intervention in moderate and severe COPD. This is particularly important at a time of financial austerity affecting healthcare services in the UK and worldwide.

Like all complex interventions, there is some debate about the optimal delivery of the programme; however, certain fundamental principles remain. The process of pulmonary rehabilitation should be preceded by a detailed and thorough assessment of the individual, to allow prescription of an individualised exercise regime but also consider other important comorbidities and the impact this may have on a successful outcome. The guideline has concentrated on a number of important aspects of pulmonary rehabilitation, including the delivery and important outcome measures; who should be referred, including consideration of chronic respiratory diseases other than COPD; structure and organisation of pulmonary rehabilitation programmes, including frequency, duration, supervision and nature of training; postexacerbation pulmonary rehabilitation; and maintenance programmes. We also consider a number of interventions and the evidence (or lack of) for their parallel delivery alongside pulmonary rehabilitation. In addition, the guideline offers some important good practice points where evidence is lacking and unlikely to become available. We complete the guideline with reference to the future audit process, important research recommendations, useful links and information on some of the practical aspects of pulmonary rehabilitation and provide a lay summary.

In several areas important to the delivery and commissioning of pulmonary rehabilitation, the evidence has allowed clearer recommendations to maintain quality and offering pulmonary rehabilitation to the right populations. In previous guidance, pulmonary rehabilitation has been reserved for those with significant disability whereas the evidence now supports extending the scope of practice to embrace those with chronic respiratory disease and with a Medical Research Council (MRC) dyspnoea score of 2 . The value in milder disease is of 
course one of restoration of function but may also take on a 'preventative role' in these patients who have, to date, experienced less functional disruption as a consequence of their COPD. However, individuals with a milder MRC score are more likely to be in full-time work and may be less able to attend traditional courses, which brings its own challenges. The frequency of supervised sessions was reviewed and there was no robust literature to support once-weekly pulmonary rehabilitation. Therefore the recommendation based on expert opinion and supported by the nature of the clinical trials demonstrating the clinical benefit of pulmonary rehabilitation is a minimum of twice-weekly supervised sessions. ${ }^{3}$ There is broad acknowledgement that strength and endurance training should be offered in rehabilitation programmes. However, the data retrieved could not identify any additional benefit of interval training, compared with continuous aerobic training, in patients with COPD, which is probably good news for most rehabilitation programmes as the level of supervision and equipment required for interval training can be a challenge.

Around the time of the 2001 BTS statement the challenge was availability, with only around $40 \%$ of hospitals offering pulmonary rehabilitation. This challenge has, in the main, been addressed as evidenced by the 2008 national COPD audit which showed $90 \%$ of units offered pulmonary rehabilitation, albeit there were concerns about access for all patients in a third of hospitals. ${ }^{5}$ In the current era of financial constraint, accessibility to pulmonary rehabilitation needs to be monitored carefully; however quality of the programme is paramount. It is hoped this guideline will contribute to improving the quality of pulmonary rehabilitation and to this end the BTS will produce quality standards for pulmonary rehabilitation to follow the publication of this guideline. Ultimately every programme should be regularly benchmarking its patient level outcomes to ensure participants gain the benefits evidenced in clinical trials. In addition, commencement and completions rates may represent useful metrics of the referral process and programme efficacy.

New challenges are prompted by the recommendations. Continuing exercise post-pulmonary rehabilitation dovetails with national strategies to promote healthy living, which includes regular exercise. Anecdotally, this ongoing maintenance exercise is offered in a variety of ways: within traditional pulmonary rehabilitation programmes; in separate 'veterans' programmes; in local leisure centres and gyms; and among British Lung Foundation Breathe Easy self-help groups. Yet there is a need for research as to what strategy is optimal and how they compare to repeat pulmonary rehabilitation programmes, which do benefit patients. The benefit of pulmonary rehabilitation is likely to extend to groups with different respiratory conditions and the-albeit limited-evidence for some of these groups is reviewed in the guideline. However, much more research is needed to determine whether training for these groups should differ and also to consider different educational elements. Evidence for other aspects of pulmonary rehabilitation may be harder to judge but seems essential to enhance programme success, for example, enthusiasm by the team disperses to the group and is a vital ingredient.

In an era of patient support groups, people with chronic respiratory disease are rightly expecting pulmonary rehabilitation. The onus is now for us to provide sufficient access to and deliver a quality pulmonary rehabilitation programme to address and optimise patient symptoms.

Competing interests None.

Provenance and peer review Not commissioned; internally peer reviewed.

\section{REFERENCES}

1 British Thoracic Society. British Thoracic Society pulmonary rehabilitation guideline. Thorax 2013;68(Supp2):1-30.

2 British Thoracic Society Standards of Care Subcommittee on Pulmonary Rehabilitation. Pulmonary rehabilitation. Thorax 2001;56:827-34.

3 Lacasse Y, Goldstein R, Lasserson TJ, et al. Pulmonary rehabilitation for chronic obstructive pulmonary disease. Cochrane Database Syst Rev 2006;(4):CD0037993. doi:10.1002/14651858.CD003793.pub2

4 Griffiths TL, Phillips CJ, Davies S, et al. Cost effectiveness of an outpatient multidisciplinary pulmonary rehabilitation programme. Thorax 2001;56:779-84.

5 Yohannes A, Stone R, Lowe D, et al. Pulmonary rehabilitation in the United Kingdom. Chron Respir Dis 2011;8:193-9. 